\title{
Helicobacter pylori enhances CIP2A expression and cell proliferation via JNK2/ATF2 signaling in human gastric cancer cells
}

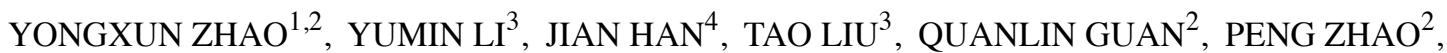 \\ LINGYUN GUO $^{5}$, KAIKUN LIU ${ }^{1}$ and DONGQIANG HE
}

\begin{abstract}
${ }^{1}$ The Second Clinical Medical School of Lanzhou University, Lanzhou, Gansu 730030; ${ }^{2}$ Department of Surgical Oncology,
The First Hospital of Lanzhou University, Lanzhou, Gansu 730000; ${ }^{3}$ Department of General Surgery,

The Second Hospital of Lanzhou University, Key Laboratory of Digestive System Tumors of Gansu Province, Lanzhou, Gansu 730030; ${ }^{4}$ Institute of Pathogenic Biology, School of Basic Medical Science, Lanzhou University, Lanzhou, Gansu 730000; ${ }^{5}$ Department of General Surgery, The Second Hospital of Lanzhou University, Lanzhou, Gansu 730030, P.R. China
\end{abstract}

Received October 7, 2013; Accepted December 24, 2013

DOI: $10.3892 /$ ijmm.2014.1615

\begin{abstract}
Helicobacter pylori (H. pylori) infection plays an important role in the development of gastric carcinomas. Cancerous inhibitor of protein phosphatase 2A (CIP2A) is a novel human oncoprotein that functions as an important regulator of cell growth and malignant transformation. In the present study, we aimed to investigate the potential mechanisms by which $H$. pylori upregulates the expression of CIP2A and the functional impact of $H$. pylori-induced CIP2A in gastric cancer cells. We demonstrated that infection of MKN-45 cells with $H$. pylori led to a marked increase in the expression of CIP2A at the mRNA and protein levels. H. pylori-induced CIP2A was associated with increased cell proliferation. In addition, $H$. pylori was found to activate the JNK2 pathway. Importantly, both $H$. pylori-induced CIP2A production and cell proliferation were partially reversed by inhibition of JNK2 signaling. Similarly, the blockade of H. pylori-induced CIP2A expression by siRNA against CIP2A also inhibited cell proliferation. Thus, $H$. pylori appears to
\end{abstract}

Correspondence to: Professor Yumin Li, Department of General Surgery, The Second Hospital of Lanzhou University, Key Laboratory of Digestive System Tumors of Gansu Province, Lanzhou, Gansu 730030, P.R. China

E-mail: liym@lzu.edu.cn

Abbreviations: CagA, cytotoxin-associated gene A; CIP2A, cancerous inhibitor of protein phosphatase $2 \mathrm{~A}$; PP2A, protein phosphatase 2A; CFU, colony-forming units; ERK1/2, extracellular signal-regulated kinases 1 and 2; FBS, fetal bovine serum; H. pylori, Helicobacter pylori; JNK, c-Jun/SAPK N-terminal kinase; ATF2, activating transcription factor 2; MAPK, mitogen-activated protein kinases; RNAi, RNA interference

Key words: Helicobacter pylori, gastric cancer, CIP2A, JNK2, proliferation, RNA interference stimulate the expression of CIP2A and proliferation of gastric cancer cells via JNK2 signaling. These findings suggest that H. pylori-induced upregulation of CIP2A contributes to the development and progression of gastric cancer. Further in vivo studies are warranted to explore the biological role of CIP2A and its interaction with JNK2 signaling in gastric cancer.

\section{Introduction}

Gastric carcinoma remains one of the most common and lethal malignancies worldwide. With approximately one million cases diagnosed annually, gastric cancer is one of the leading causes of cancer-related mortality worldwide (1). Helicobacter pylori $(H$. pylori) has been defined as a class I carcinogen by the World Health Organization (2), and its persistent colonization in the stomach significantly increases the risk of gastric adenocarcinoma (3). During the course of $H$. pylori infection, bacterial virulence factors, such as cytotoxin-associated gene A (CagA), interact with numerous signaling molecules and elicit a series of cellular events; one of which is the $H$. pylori-induced activation of mitogen-activated protein kinases (MAPK) which has been implicated in the development of gastric adenocarcinoma $(4,5)$.

MAPK consists of a family of ubiquitously expressed proteins including extracellular signal-regulated kinases 1 and $2($ ERK1/2), p38 and c-Jun/SAPK N-terminal kinases (JNK) $(6,7)$. In addition to activating MAPK, H. pylori infection also induces the expression of other inflammatory responsive genes including nuclear factor- $\kappa \mathrm{B}(\mathrm{NF}-\kappa \mathrm{B})(8)$, cyclooxygenase-2 (COX-2) (9) and aquaporin 3 (AQP3) (10). $H$. pylori has been reported to stimulate the proliferation of gastric epithelial cells both in vitro and in vivo $(5,11,12)$ and to alter intercellular tight junctions (13), thus facilitating the malignant transformation of the gastric mucosa.

Protein phosphatase 2A (PP2A) facilitates the proteolytic degradation of oncoprotein Myc and prevents malignant cell growth (14). Cancerous inhibitor of protein 
phosphatase 2A (CIP2A) is a recently identified human oncoprotein that stabilizes c-Myc protein by inhibiting its degradation. CIP2A has been reported to promote the proliferation of various types of cancer cells (15). Moreover, CIP2A is required for malignant cell growth and the malignant transformation of human cells (15). Overexpression of CIP2A has been observed in many types of solid tumors such as head and neck squamous cell carcinoma and gastric cancer $(15,16)$. In human gastric cancer, a higher level of CIP2A was noted in cancerous tissues when compared to the level in non-cancerous mucosa, suggesting that CIP2A plays an oncogenic role in the development of human gastric cancer $(16,17)$. Previous research has shown that JNK2 is a key regulator of CIP2A expression (18).

Persistent $H$. pylori infection is a well-recognized condition that is closely linked to the development of gastric cancer. It has been shown that $H$. pylori infection upregulates the expression of CIP2A in gastric cancer cells (13). CagA, a major virulent factor of $H$. pylori, activates the JNK pathway and is thus involved in promoting gastric cancer progression $(4,19)$. In addition, JNK 2 was found to regulate CIP2A expression via activation of transcription factor 2 (ATF2) (18). However, it is not clear whether JNK2 is involved in the regulation of CIP2A expression following $H$. pylori infection.

In the present study, we aimed to examine the effect of H. pylori infection on JNK2 and CIP2A and the subsequent biological impact on gastric cancer cells. We found that $H$. pylori increased CIP2A expression and promoted cell proliferation via the JNK2/ATF2 signaling pathway in MKN-45 cells. Possible implications of CIP2A and its interaction with JNK signaling in gastric cancer are discussed.

\section{Materials and methods}

Cell culture, RNA interference (RNAi) and reagents. Human gastric cancer cell line MKN-45 (Chinese Academy of Sciences, Shanghai, China) was cultured in RPMI-1640 (HyClone Laboratories, Inc., Logan, UT, USA) supplemented with $10 \%$ fetal bovine serum (FBS) (HyClone Laboratories, Inc.) and $1 \%$ penicillin and streptomycin (North China Pharmaceutical Co., Inc., Shijiazhuang, China) in a humidified atmosphere containing $5 \% \mathrm{CO}_{2}$ at $37^{\circ} \mathrm{C}$. The specific siRNA against CIP2A and the scrambled control siRNA were purchased from Invitrogen (Carlsbad, CA, USA). The siRNA sequence for CIP2A was 5'-GACAACUGUCAAGUGUACCACU CUU-3' (16). Lipofectamine ${ }^{\mathrm{TM}} 2000$ was used to transfect the siRNA sequences into MKN-45 cells according to the manufacturer's instructions (Invitrogen). SP600125, a JNK2 inhibitor, was purchased from InvivoGen (San Diego, CA, USA).

H. pylori culture and co-culture with MKN-45 cells. The H. pylori strains used in this study were CagA and VacA positive standard strain (NCTC11637) (Institute of Pathogenic Biology, School of Basic Medical Science, Lanzhou University, Lanzhou, China). H. pylori were cultured on Columbia agar plates (Difco Laboratories, Detroit, MI, USA) containing 5\% sheep blood at $37^{\circ} \mathrm{C}$ for $72 \mathrm{~h}$ under microaerophilic conditions using an anaerobic box (Mitsubishi Gas Chemical Co., Inc., Tokyo, Japan). The bacteria were harvested and resuspended in antibiotic-free RPMI-1640 medium supplemented with $10 \%$
FBS. The density of the bacteria was adjusted by measuring the optical density (OD) at $600 \mathrm{~nm}$, in which one unit of $\mathrm{OD}_{600}$ corresponds to $1 \times 10^{8}$ colony-forming units $(\mathrm{CFU}) / \mathrm{ml}$. For the cell and bacterial co-culture, MKN-45 cells were cultured in RPMI-1640 medium in the presence of $H$. pylori at a cell-tobacterium ratio of 1:100.

Quantitative real-time reverse transcription-polymerase chain reaction ( $q R T-P C R)$ assay. Total RNA from the cells was prepared using RNAiso Plus reagent (Takara Biotechnology Co., Dalian, China) under an RNase-free condition, and cDNA was synthesized using the Primescript ${ }^{\mathrm{TM}}$ Reverse Transcription (RT) Master Mix (Takara Biotechnology Co.) according to the manufacturer's protocols. Reverse transcription reaction was performed at $37^{\circ} \mathrm{C}$ for $15 \mathrm{~min}$ followed by $85^{\circ} \mathrm{C}$ for $5 \mathrm{sec}$. qRT-PCR amplifications were performed with the Applied Biosystems 7500/7500 Fast Real-Time PCR Software (Applied Biosystems) using the SYBR ${ }^{\circledR}$ Premix Ex Taq ${ }^{\mathrm{TM}}$ II (Takara Biotechnology Co.). Reactions were carried out in a $20-\mu 1$ reaction volume following the manufacturer's instructions. The thermal cycle conditions were as follows: $95^{\circ} \mathrm{C}$ for $30 \mathrm{sec}$, followed by 40 cycles of $95^{\circ} \mathrm{C}$ for $5 \mathrm{sec}$ and $60^{\circ} \mathrm{C}$ for $34 \mathrm{sec}$. GAPDH was used as an internal control. The primer sequences were: CIP2A sense, 5'-GGCACTTGGAGGTAATTTCT-3' and antisense, 5'-CTGGTTTCAATGTCTACTGCTAG-3' (GenBank accession no. NM020890); GAPDH sense, 5'-AAG GCTGGGGCTCATTTG-3' and antisense, 5'-AGGAGGCA TTGCTGATGATC-3' (GenBank accession no. NM002046). All primers were synthesized by Takara Biotechnology Co. The mRNA expression level was expressed as relative expression to the basal level without $H$. pylori stimulation. Data were analyzed according to the comparative $\mathrm{Ct}$ method.

Western blot assay. Treated cells were washed with ice-cold phosphate-buffered saline (PBS) and then lysed using RIPA lysis buffer (Beyotime Biotechnology, Haimen, China) supplemented with $1 \mathrm{mmol} / \mathrm{l}$ phenylmethanesulfonyl fluoride (PMSF). After centrifugation at 12,000 rpm for $15 \mathrm{~min}$ at $4^{\circ} \mathrm{C}$, the supernatant was collected and the protein concentration was measured by the BCA protein assay (Beyotime Biotechnology). An equal amount of protein $(50 \mu \mathrm{g})$ from each sample was separated on a $10 \%$ SDS polyacrylamide gel and transferred onto polyvinylidene fluoride (PVDF) membranes. The membranes were blocked with $5 \%$ nonfat dry milk in Tris-buffered saline containing $0.1 \%$ Tween-20 (TBST) for $2 \mathrm{~h}$ at room temperature and incubated with the respective primary antibodies overnight at $4{ }^{\circ} \mathrm{C}$. The following primary antibodies were used for the procedure (all diluted at 1:1,000 in TBST): anti-CIP2A (2G10-3B5) (Santa Cruz Biotechnology, Inc., Santa Cruz, CA, USA), anti-phospho-ATF2 and anti-ATF2 (Cell Signaling Technology, Inc., Danvers, MA, USA), antiphospho-JNK2 (G-7) and anti-JNK2 (G-7) (Santa Cruz Biotechnology, Inc.) and anti- $\beta$-actin (Zhongshan Golden Bridge Biotech, Beijing, China). $\beta$-actin was used as a loading control. After overnight incubation, the membranes were washed three times with TBST, and then incubated with the horseradish peroxidase-conjugated secondary antibodies (Zhongshan Golden Bridge Biotech) (1:10,000) at room temperature for $1 \mathrm{~h}$. The signals were detected using the SuperSignal West Pico Chemiluminescent Substrate (Thermo 

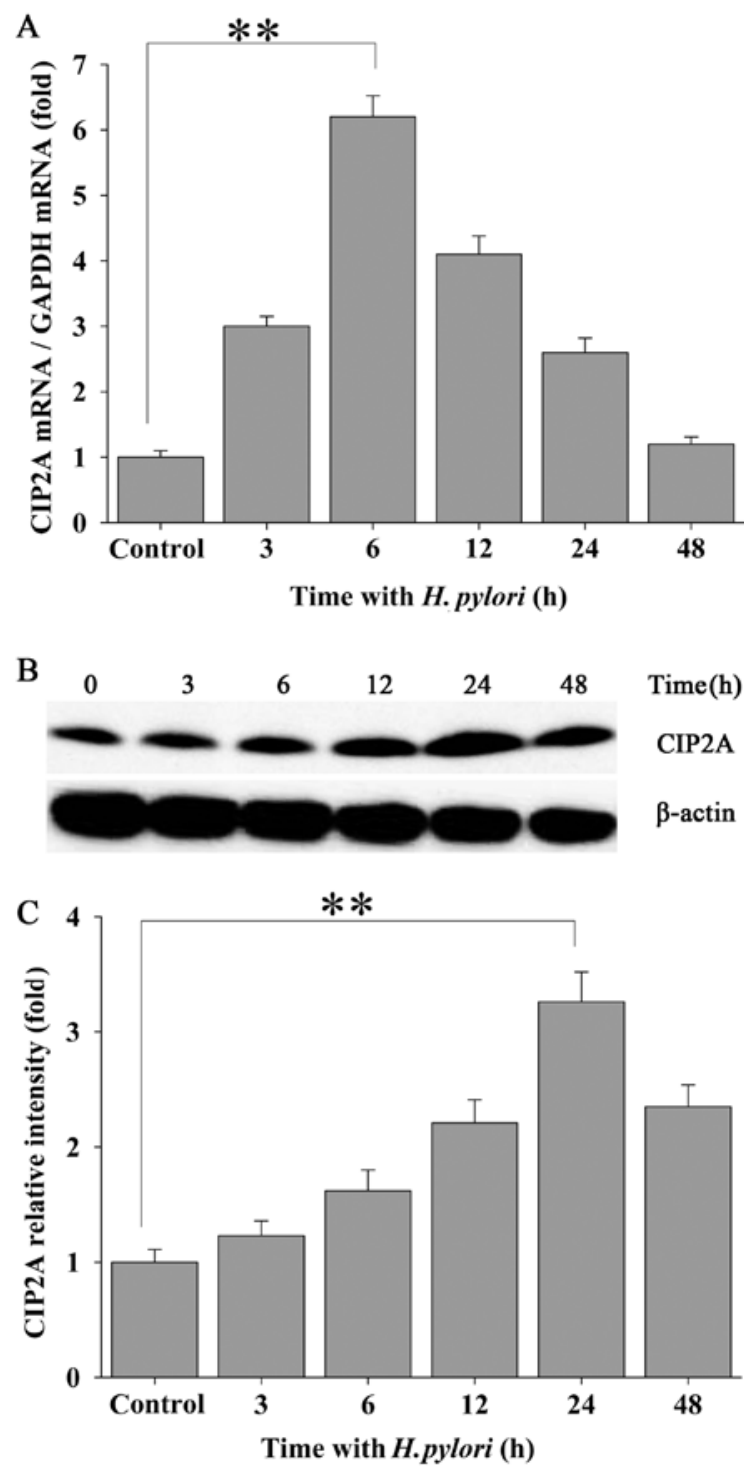

Figure 1. Effect of $H$. pylori on the expression of CIP2A in MKN-45 cells. (A) Infection of cells by $H$. pylori led to an upregulation of CIP2A at the mRNA level, which peaked at $6 \mathrm{~h}$ following bacterial infection and then gradually declined while still maintaining an elevated level until $48 \mathrm{~h}$ (B) Increased CIP2A expression was also confirmed at the protein level. The CIP2A protein expression was analyzed by western blotting using the whole cell lysate harvested following $H$. pylori infection at different time-points, and $(\mathrm{C})$ the results were normalized and quantified against the expression of $\beta$-actin, which peaked at $24 \mathrm{~h}$ following bacterial infection. The results represent the mean \pm standard deviation (SD) of three independent experiments. ${ }^{* *} \mathrm{P}<0.01$, compared with the untreated (control) group.

Fisher Scientific, Inc., Rockford, IL, USA) and imaged using a VersaDoc Imaging System (Bio-Rad Laboratories Co., Ltd. Hercules, CA, USA). Data obtained from the western blotting experiments were analyzed by Bio-Rad Quantity One Software v4.62 (Bio-Rad Laboratories Co., Ltd.).

MTT assay. Cell proliferation was measured using the dimethylthiazolyl-2,5-diphenyltetrazolium bromide (MTT) assay. Cells were plated at a density of 5,000 cells/well in 96-well plates in $200 \mu \mathrm{l}$ antibiotic-free culture medium and cultured for $12 \mathrm{~h}$. After the appropriate treatment, MTT reagent was added ( $20 \mu \mathrm{l} /$ well of $5 \mathrm{~g} / 1$ solution in PBS) into each well. Subsequently, incubation was carried out at $37^{\circ} \mathrm{C}$ for $4 \mathrm{~h}$ and then washing with PBS. Next, $200 \mu \mathrm{l}$ dimethyl sulfoxide (DMSO) was added to each well. The absorbance was measured at $490 \mathrm{~nm}$. All samples were assayed repeatedly in 6 wells. The cell proliferation rate was quantified as the fraction of cells surviving relative to the untreated controls.

Colony formation assay. Anchorage-independent growth was measured by colony formation assay. MKN-45 cells were transfected with siRNA against CIP2A or scrambled siRNA for $72 \mathrm{~h}$. The transfected cells were infected with or without $H$. pylori for $3 \mathrm{~h}$ and planted into 6-well plates at low density (300 cells/well). After 14 days, cells were fixed and stained with crystal violet. Foci and colonies containing $>50$ cells were counted using a microscope.

Statistical analysis. All experiments were repeated three times, and data are expressed as mean \pm standard deviation (SD). Statistical significance was determined with one-way ANOVA followed by the Bonferroni correction. All statistical analyses were performed using SPSS 19.0 (IBM, Armonk, NY, USA), and data values were displayed using SigmaPlot 10.0 (Systat Software Inc., Chicago, IL, USA). A P-value of $<0.05$ was considered to indicate a statistically significant result.

\section{Results}

H. pylori upregulates $C I P 2 A$ expression in $M K N-45$ cells. To investigate the effects of $H$. pylori infection on CIP2A expression in human gastric carcinoma cells, confluent MKN-45 cells were incubated with $H$.pylori at a density of 100 bacteria per cell for $0,3,6,12,24$ and $48 \mathrm{~h}$. qRT-PCR assays were performed to examine the mRNA transcript levels of CIP2A in the MKN-45 cells. Treatment of the MKN-45 cells with H. pylori led to a significantly elevated expression of CIP2A at the mRNA level which peaked at $6 \mathrm{~h}$ of incubation and declined thereafter while still maintaining an elevated level (Fig. 1A). Increased expression of CIP2A was also confirmed at the protein level by western blot analysis (Fig. 1B and C), and this level peaked at $24 \mathrm{~h}$ following bacterial infection.

The JNK2 signaling pathway is involved in H. pyloriregulated CIP2A expression in $M K N-45$ cells. To ascertain whether JNK2 signaling is involved in the $H$. pylori-induced expression of CIP2A, the expression of phosphorylated JNK2 (p-JNK2) in response to H. pylori infection was evaluated by western blot analysis using a phospho-specific antibody against JNK2. Approximately 20 min after MKN-45 cells were challenged with $H$. pylori, a steady increase in the expression of p-JNK2 was noted, which exhibited an approximate time-dependent manner, whereas total JNK2 remained unchanged (Fig. 2A and C). To further confirm the H. pyloriinduced activation of the JNK2 pathway in MKN-45 cells, we evaluated the expression of phospho-ATF2 (p-ATF2), a substrate for p-JNK2. Activation of the JNK2 pathway was followed by a transient increase in the expression of p-ATF2 in the H.pylori-treated MKN-45 cells (Fig. 2A, B, D and E).

Inhibition of JNK2 attenuates $H$. pylori-stimulated CIP2A expression in MKN-45 cells. To further ascertain whether the H.pylori-induced upregulation of CIP2A is mediated through 

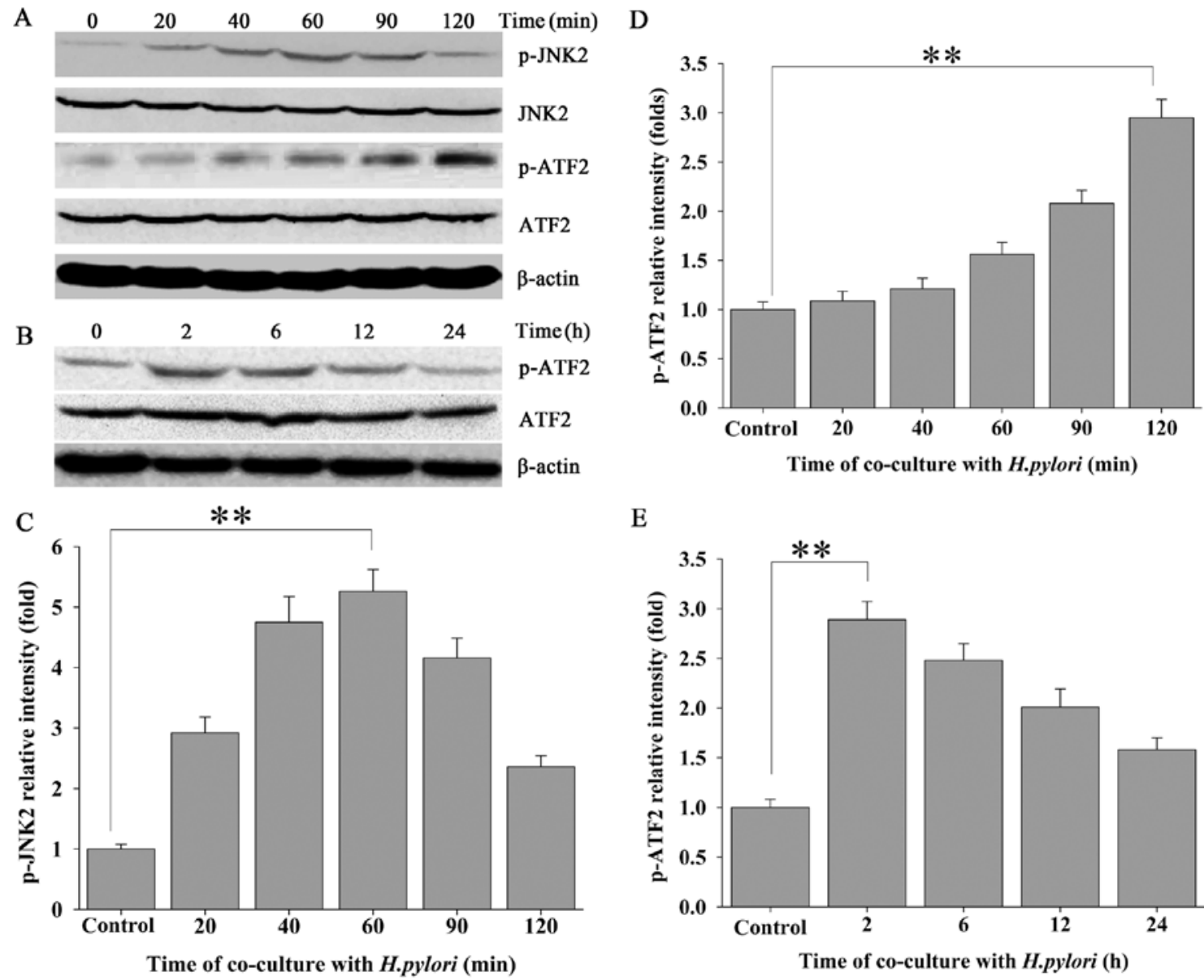

Figure 2. Effect of $H$. pylori infection on JNK2 signaling in MKN-45 cells. MKN-45 cells were cultured in the presence of $H$. pylori at a density of 1:100. (A) The expression level of phosphorylated JNK2 (p-JNK2) was detected by western blot analysis, and (C) the results were quantitatively analyzed by densitometry. (A and B) Activation of ATF2, a downstream target of the JNK2 pathway, was also examined by western blot analysis using its specific phosphoantibody, and (D and E) the results were quantitatively analyzed by densitometry. Blots shown are representative of three separate experiments with similar results. ${ }^{* *} \mathrm{P}<0.01$.

the JNK2 pathway, MKN-45 cells were pretreated with $20 \mu \mathrm{M}$ of the JNK2 inhibitor SP600125 for $2 \mathrm{~h}$ followed by treatment with $H$. pylori. Inhibition of JNK2 signaling by SP600125 led to a significant but transient reduction in CIP2A mRNA in response to $H$. pylori infection (Fig. 3A). These changes were also reflected at the protein level (Fig. 3B and C).

$H$. pylori induces proliferation of $M K N-45$ cells via CIP $2 A$. In order to examine the functional impact of $H$. pylori-induced upregulation of CIP2A in gastric cancer cells, we utilized siRNA techniques to modulate the expression of CIP2A and examined the effect on cell proliferation. MKN-45 cells were transfected with siRNA against CIP2A or scrambled siRNA, and the effect on the proliferation of MKN-45 cells was examined by MTT assay. As expected, siRNA against CIP2A not only significantly knocked down the basal level of CIP2A expression at the mRNA (by 90\%) (Fig. 4A) and protein (by 82\%) (Fig. 4B and C) levels, as determined by qRT-PCR and western blot analysis, respectively, but also the $H$. pylori-induced upregulation of CIP2A at the protein level (Fig. 4D and E). Parallel to these changes, $H$. pylori-induced proliferation (Fig. 5A) and colony formation (Fig. 5B and C) of the MKN-45 cells were significantly attenuated by CIP2A knockdown. These data suggest that CIP2A is involved in H. pylori-induced proliferation of MKN-45 cells.

H. pylori induces the proliferation of $M K N-45$ cells via $J N K 2$ signaling. The results as reported above showed that $H$. pylori-induced upregulation of CIP2A in MKN45 cells was regulated via JNK2 signaling, and that $H$. pylori-induced proliferation of MKN45 cells was mediated by CIP2A. Here, we further investigated whether JNK2 signaling is involved in $H$. pylori-induced proliferation of MKN- 45 cells. MKN-45 cells were treated with the JNK2 inhibitor SP600125 for $2 \mathrm{~h}$ prior to $H$. pylori infection. Cell proliferation was examined by the MTT assay. Treatment of MKN-45 cells with SP600125 attenuated the H.pylori-stimulated cell proliferation (Fig. 6). These data indicate that $H$. pylori-induced proliferation of MKN-45 cells is dependent upon JNK2 signaling.

\section{Discussion}

H. pylori infection is the major risk factor for gastric cancer, which is the second leading cause of cancer-related mortality worldwide (20). The prevalence of $H$. pylori infection varies according to geographical region, race, age and socioeconomic 
A

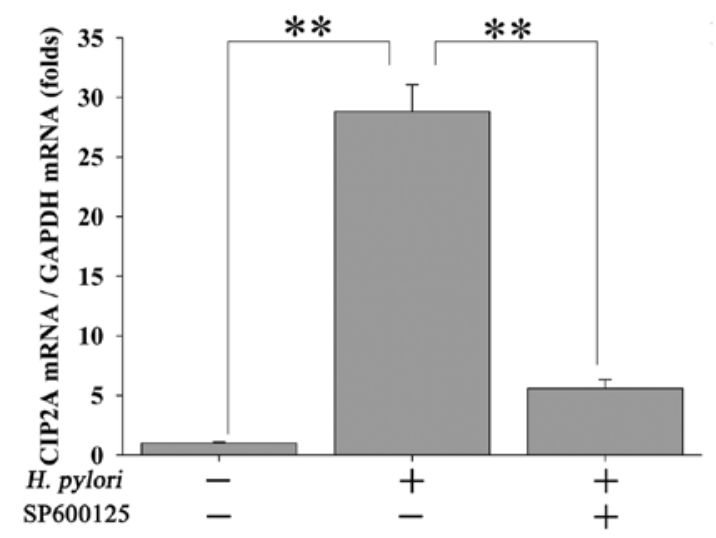

B

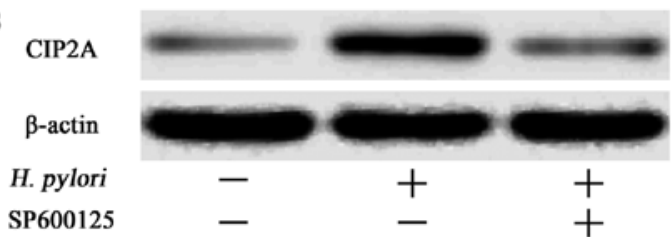

C

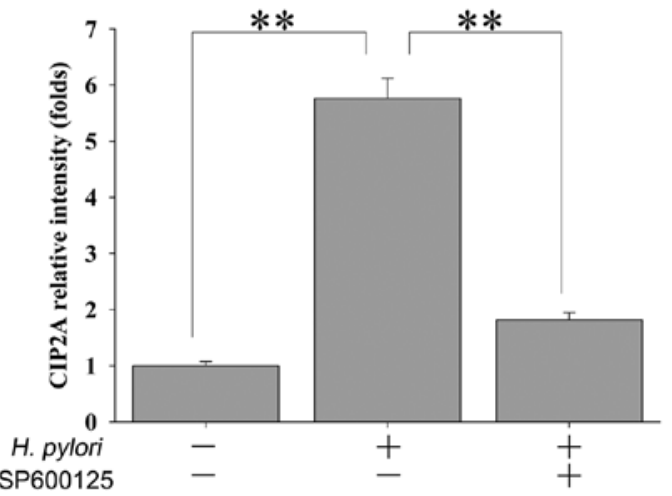

Figure 3. Effect of the JNK2 inhibitor on $H$. pylori-induced CIP2A expression in MKN-45 cells. Cultured MKN-45 cells were pretreated with the JNK2 inhibitor SP600125 $(20 \mu \mathrm{M})$ for $2 \mathrm{~h}$, followed by incubation with $H$. pylori as described in Materials and methods. (A) The expression of CIP2A mRNA was detected by quantitative real-time reverse transcription-polymerase chain reaction (qRT-PCR) at $6 \mathrm{~h}$ following addition of $H$. pylori. Inhibition of JNK2 signaling almost abolished $\mathrm{H}$. pylori-induced upregulation of CIP2A. (B) This finding was further verified at the protein level by western blot analysis, and (C) the results were analyzed quantitatively at $24 \mathrm{~h}$ after addition of $H$. pylori. The results represent the mean \pm standard deviation (SD) of three independent experiments. Representative blots are shown. ${ }^{* *} \mathrm{P}<0.01$.

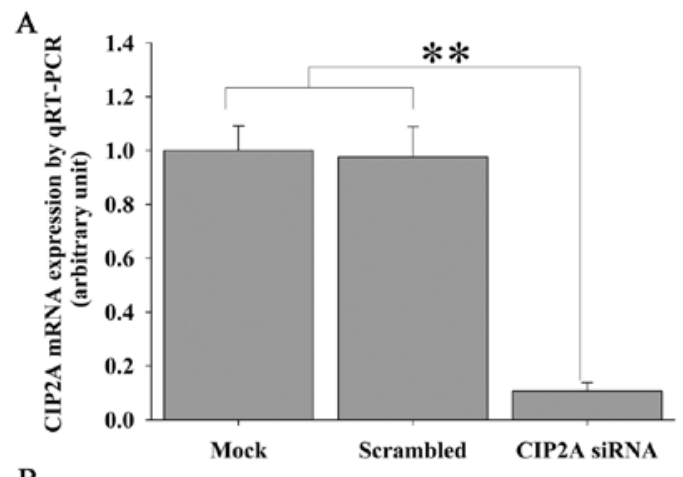

D

B

\section{CIP2A \\ $\beta$-actin}

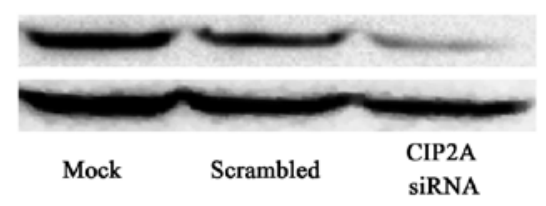

$\mathrm{C}$

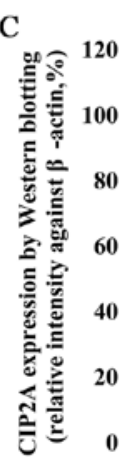

$* *$

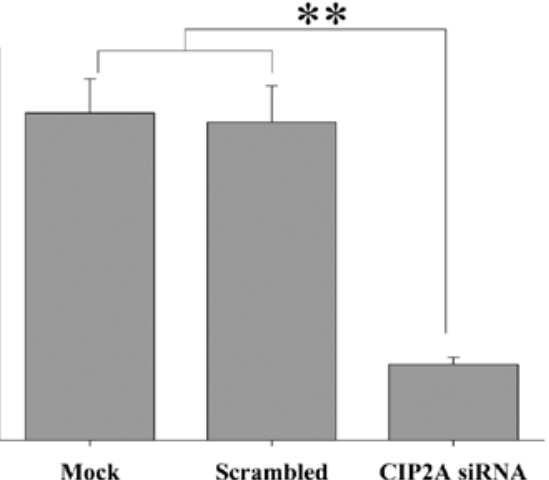

CIP2A

$\beta$-actin

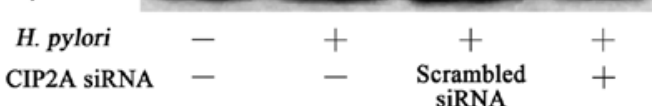

$\mathrm{E}$

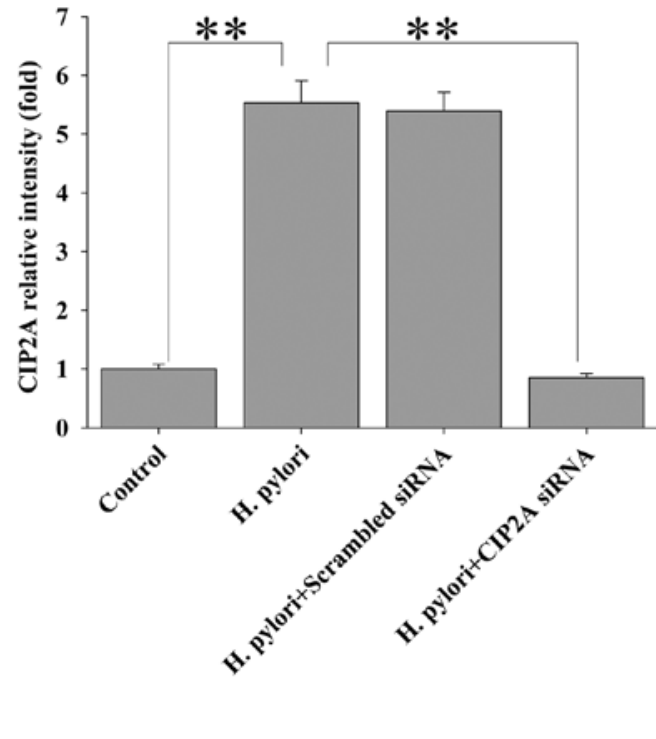

Figure 4. siRNA against CIP2A successfully knocked down the expression of CIP2A in MKN-45 cells. MKN-45 cells were treated with scrambled siRNA or CIP2A-specific siRNA for $48 \mathrm{~h}$. The expression of CIP2A was examined by (A) quantitative real-time reverse transcription-polymerase chain reaction (qRT-PCR) and (B) verified at the protein level by western blotting and (C) the results were quantified by densitometry. Furthermore, knockdown of CIP2A by its specific siRNA was able to reverse the H. pylori-stimulated upregulation of CIP2A, as demonstrated by (D) western blot analysis, and (E) the results were quantitatively analyzed by densitometry. The data are expressed as the mean \pm standard deviation (SD) of three independent experiments. ${ }^{* *} \mathrm{P}<0.01$. 

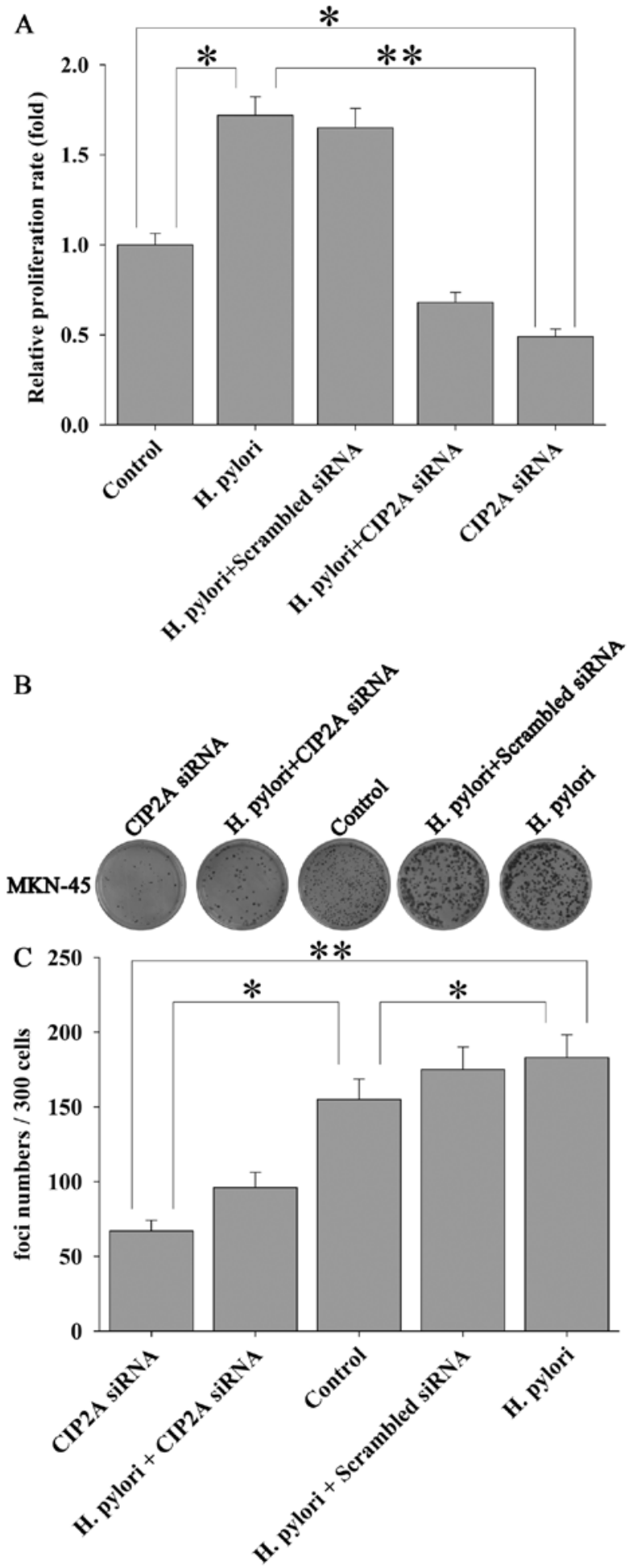

Figure 5. Knockdown of CIP2A by siRNA effectively inhibits the $H$. pyloriinduced proliferation and colony formation of MKN-45 cells. MKN-45 cells were transfected with scrambled siRNA or CIP2A siRNA for $48 \mathrm{~h}$ in the presence or absence of $H$. pylori for $24 \mathrm{~h}$, as described in Materials and methods. (A) As measured by MTT assay, knockdown of CIP2A successfully blocked the H. pylori-induced cell proliferation. (B) In addition, knockdown of CIP2A led to a marked reduction in the colony forming units (CFU), and (C) the results are quantitatively presented. The data are expressed as the mean \pm standard deviation (SD) of three independent experiments. ${ }^{*} \mathrm{P}<0.05$; ${ }^{* *} \mathrm{P}<0.01$.

status (21). A high prevalence of $H$. pylori infection is present in several regions of China such as Hexi Corridor where the

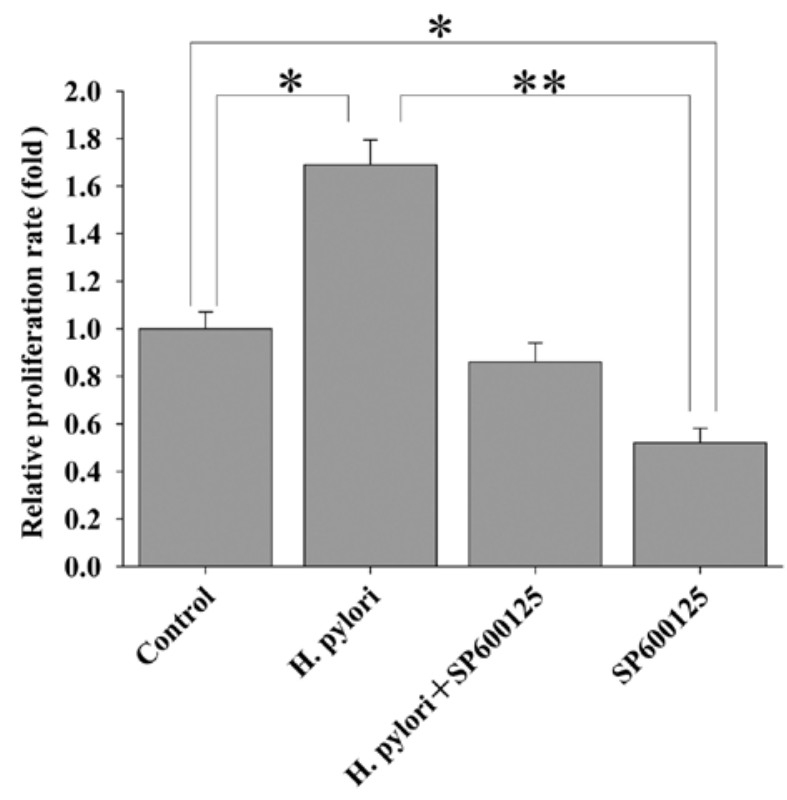

Figure 6. $H$. pylori-induced proliferation of MKN-45 cells is dependent on JNK2 signaling. Cells were treated with SP600125 $(20 \mu \mathrm{M})$ for $2 \mathrm{~h}$, followed by $H$. pylori infection for $24 \mathrm{~h}$, as described in Materials and methods, and the cell proliferation was determined using the MTT assay. Inhibition of JNK2 signaling markedly reduced basal and $H$. pylori-induced cell proliferation. The data are expressed as the mean \pm standard deviation (SD) of three independent experiments. ${ }^{*} \mathrm{P}<0.05 ;{ }^{* *} \mathrm{P}<0.01$.

incidence of gastric cancer is high (22). Clinical epidemiological data has clearly shown a strong association between $H$. pylori infection and gastric carcinogenesis (23-25), and eradication of $H$. pylori was found to reduce the incidence of gastric cancer $(26,27)$.

CIP2A was reported to promote cell proliferation and tumor formation by stabilizing the oncogene c-Myc (15-17). Overexpression of CIP2A has been shown in human gastric carcinoma tissues $(16,17)$. A recent study revealed an association between $H$. pylori infection and CIP2A expression (13); yet, the biological impact of $H$. pylori-induced CIP2A expression and its underlying molecular mechanisms are not clearly defined.

It has been reported that $H$. pylori infection activates the three major components of MAPK signaling in gastric epithelial cells, namely ERK1/2, p38 and JNK $(4,28,29)$. The MAPK pathway plays a crucial role in mediating a number of events in host cell physiology, including gene transcription, mitosis, motility, adhesion, metabolism and apoptosis $(6,7)$. The JNK-mediated cascade is the basic signal transduction pathway that regulates essential cellular processes $(30,31)$. It has been established that $H$. pylori induces gene expression in human gastric epithelial cells by activating JNK signaling pathways (29,32-34). Among the JNK family, JNK2 is essential for Ras signaling-induced cellular transformation of human epithelial cells $(35,36)$.

Previous studies have shown that JNK2 is essential for the expression of CIP2A, and JNK2 regulates CIP2A transcription via ATF2 (18). In the present study, we further demonstrated that $H$. pylori infection significantly increased the expression of CIP2A in MKN-45 cells, both at the mRNA and protein 
levels. In addition, $H$. pylori-induced CIP2A required functional JNK2, as inhibition of JNK2 by its inhibitor SP600125 significantly decreased the expression of CIP2A in response to $H$. pylori. Thus, JNK2 signaling plays an essential role in H. pylori-induced CIP2A expression in gastric cancer cells.

Perhaps the most important implication of these finding is the functional impact of CIP2A and JNK2 on gastric epithelial cells during $H$. pylori infection. It is known that both CIP2A and $H$. pylori infection enhance cell proliferation $(15,16,37$ 39). In the present study, blocking of CIP2A by its specific siRNA significantly attenuated $H$. pylori-induced cell proliferation, suggesting that $H$. pylori induced the proliferation of gastric epithelial cells through upregulation of CIP2A. This mechanism warrants further investigation to ascertain whether CIP2A is suitable for use as a therapeutic target for gastric cancer. As our study was conducted using a single cell line, further studies using multiple cell lines, particularly using normal gastric mucosa cells are needed, and appropriate in vivo studies must be performed to further verify the findings reported in the present study.

In conclusion, our results demonstrated that $H$. pylori infection promotes MKN-45 gastric epithelial cell proliferation through upregulation of CIP2A expression, and this was likely mediated via the JNK2 signaling pathway. The data presented here suggest that CIP2A plays a critical role in the development of human gastric carcinoma, elucidating another mechanism of $H$. pylori-induced carcinogenesis. Further studies, particularly in vivo studies using appropriate animal models, are needed to verify whether the interaction between CIP2A and JNK2 signaling is indeed essential for the development of $H$. pylori-induced gastric cancer formation, and if so, what the potential is for possible gene therapy for this malignancy.

\section{Acknowledgements}

This study was supported by the Scientific Research Funds of the Health Sector Projects in Gansu Province (grant no. GWGL2010-17), the National Natural Science Foundation of China (grant no. 31270532) and the Fundamental Research Funds of the Central Universities, China (grant no. lzujbky-2013-m04).

\section{References}

1. Jemal A, Bray F, Center MM, Ferlay J, Ward E and Forman D: Global cancer statistics. CA Cancer J Clin 61: 69-90, 2011.

2. Konturek PC, Konturek SJ and Brzozowski T: Helicobacter pylori infection in gastric cancerogenesis. J Physiol Pharmacol 60: 3-21, 2009.

3. Polk DB and Peek RM Jr: Helicobacter pylori: gastric cancer and beyond. Nat Rev Cancer 10: 403-414, 2010.

4. Ding SZ, Smith MF Jr and Goldberg JB: Helicobacter pylori and mitogen-activated protein kinases regulate the cell cycle, proliferation and apoptosis in gastric epithelial cells. J Gastroenterol Hepatol 23: e67-e78, 2008.

5. Chen YC, Wang Y, Li JY, Xu WR and Zhang YL: H. pylori stimulates proliferation of gastric cancer cells through activating mitogen-activated protein kinase cascade. World J Gastroenterol 12: 5972-5977, 2006.

6. Johnson GL and Lapadat R: Mitogen-activated protein kinase pathways mediated by ERK, JNK, and p38 protein kinases Science 298: 1911-1912, 2002.

7. Chang L and Karin M: Mammalian MAP kinase signalling cascades. Nature 410: 37-40, 2001.
8. Brandt S, Kwok T, Hartig R, König W and Backert S: NF-kappaB activation and potentiation of proinflammatory responses by the Helicobacter pylori CagA protein. Proc Natl Acad Sci USA 102: 9300-9305, 2005.

9. Li Q, Liu N, Shen B, et al: Helicobacter pylori enhances cyclooxygenase 2 expression via p38MAPK/ATF-2 signaling pathway in MKN45 cells. Cancer Lett 278: 97-103, 2009.

10. Wang G, Gao F, Zhang W, et al: Involvement of Aquaporin 3 in Helicobacter pylori-related gastric diseases. PLoS One 7: e49104, 2012.

11. de Paulis A, Prevete N, Rossi FW, et al: Helicobacter pylori $\mathrm{Hp}(2-20)$ promotes migration and proliferation of gastric epithelial cells by interacting with formyl peptide receptors in vitro and accelerates gastric mucosal healing in vivo. J Immunol 183: 3761-3769, 2009 .

12. Nagy TA, Wroblewski LE, Wang D, et al: $\beta$-Catenin and p120 mediate PPAR $\delta$-dependent proliferation induced by Helicobacter pylori in human and rodent epithelia. Gastroenterology 141: 553-564, 2011.

13. Zhao D, Liu Z, Ding J, et al: Helicobacter pylori CagA upregulation of CIP2A is dependent on the Src and MEK/ERK pathways. J Med Microbiol 59: 259-265, 2009.

14. Seshacharyulu P, Pandey P, Datta K and Batra SK: Phosphatase: PP2A structural importance, regulation and its aberrant expression in cancer. Cancer Lett 335: 9-18, 2013.

15. Junttila MR, Puustinen P, Niemelä M, et al: CIP2A inhibits PP2A in human malignancies. Cell 130: 51-62, 2007.

16. Li W, Ge Z, Liu C, et al: CIP2A is overexpressed in gastric cancer and its depletion leads to impaired clonogenicity, senescence, or differentiation of tumor cells. Clin Cancer Res 14: 3722-3728, 2008.

17. Khanna A, Böckelman C, Hemmes A, et al: MYC-dependent regulation and prognostic role of CIP2A in gastric cancer. J Natl Cancer Inst 101: 793-805, 2009.

18. Mathiasen DP, Egebjerg C, Andersen SH, et al: Identification of a c-Jun N-terminal kinase-2-dependent signal amplification cascade that regulates c-Myc levels in ras transformation. Oncogene 31: 390-401, 2011.

19. Wandler AM and Guillemin K: Transgenic expression of the Helicobacter pylori virulence factor CagA promotes apoptosis or tumorigenesis through JNK activation in Drosophila. PLoS Pathog 8: e1002939, 2012.

20. Thun MJ, DeLancey JO, Center MM, Jemal A and Ward EM: The global burden of cancer: priorities for prevention. Carcinogenesis 31: 100-110, 2010.

21. Fock KM and Ang TL: Epidemiology of Helicobacter pylori infection and gastric cancer in Asia. J Gastroenterol Hepatol 25: 479-486, 2010.

22. Zhang DH, Zhou LY, Lin SR, et al: Recent changes in the prevalence of Helicobacter pylori infection among children and adults in high- or low-incidence regions of gastric cancer in China. Chin Med J (Engl) 122: 1759-1763, 2009.

23. Wang XQ, Yan H, Terry PD, et al: Interaction between dietary factors and Helicobacter pylori infection in noncardia gastric cancer: a population-based case-control study in China. J Am Coll Nutr 31: 375-384, 2012.

24. Zhang Y, Sun LP, Xing CZ, et al: Interaction between GSTP1 Val allele and $H$. pylori infection, smoking and alcohol consumption and risk of gastric cancer among the Chinese population. PLoS One 7: e47178, 2012.

25. Epplein M, Zheng W, Xiang YB, et al: Prospective study of Helicobacter pylori biomarkers for gastric cancer risk among Chinese men. Cancer Epidemiol Biomarkers Prev 21: 2185-2192, 2012.

26. Fukase K, Kato M, Kikuchi S, et al: Effect of eradication of Helicobacter pylori on incidence of metachronous gastric carcinoma after endoscopic resection of early gastric cancer: an open-label, randomised controlled trial. Lancet 372: 392-397, 2008.

27. Fuccio L, Zagari RM, Eusebi LH, et al: Meta-analysis: can Helicobacter pylori eradication treatment reduce the risk for gastric cancer? Ann Intern Med 151: 121-128, 2009.

28. Jang SH, Lim JW and Kim H: Beta-carotene inhibits Helicobacter pylori-induced expression of inducible nitric oxide synthase and cyclooxygenase-2 in human gastric epithelial AGS cells. J Physiol Pharmacol 60 (Suppl 7): S131-S137, 2009.

29. Lim JW and Kim H: Role of protease-activated receptor- 2 on cell death and DNA fragmentation in Helicobacter pylori-infected gastric epithelial cells. J Transl Med 8: 85, 2010. 
30. Zhao C, Ma H, Bu X, Wang W and Zhang N: SFRP5 inhibits gastric epithelial cell migration induced by macrophage-derived Wnt5a. Carcinogenesis 34: 146-152, 2013.

31. Zhong J, Zhao M, Luo Q, et al: CCDC134 is down-regulated in gastric cancer and its silencing promotes cell migration and invasion of GES-1 and AGS cells via the MAPK pathway. Mol Cell Biochem 372: 1-8, 2013.

32. Choi IJ, Fujimoto S, Yamauchi K, Graham DY and Yamaoka Y: Helicobacter pylori environmental interactions: effect of acidic conditions on $H$. pylori-induced gastric mucosal interleukin- 8 production. Cell Microbiol 9: 2457-2469, 2007.

33. Ding S-Z, Olekhnovich IN, Cover TL, Peek RM Jr, Smith MF Jr and Goldberg JB: Helicobacter pylori and mitogen-activated protein kinases mediate activator protein-1 (AP-1) subcomponent protein expression and DNA-binding activity in gastric epithelial cells. FEMS Immunol Med Microbiol 53: 385-394, 2008.

34. Matsumoto A, Isomoto $\mathrm{H}$, Nakayama $\mathrm{M}$, et al: Helicobacter pylori VacA reduces the cellular expression of STAT3 and prosurvival $\mathrm{Bcl}-2$ family proteins, $\mathrm{Bcl}-2$ and $\mathrm{Bcl}-\mathrm{XL}$, leading to apoptosis in gastric epithelial cells. Dig Dis Sci 56: 999-1006, 2011.
35. Ke H, Harris R, Coloff JL, et al: The c-Jun NH2-terminal kinase 2 plays a dominant role in human epidermal neoplasia. Cancer Res 70: 3080-3088, 2010.

36. Cellurale C, Sabio G, Kennedy NJ, et al: Requirement of c-Jun $\mathrm{NH}(2)$-terminal kinase for Ras-initiated tumor formation. Mol Cell Biol 31: 1565-1576, 2011.

37. Li N, Han L, Chen J, Lin X, Chen H and She F: Proliferative and apoptotic effects of gastric epithelial cells induced by coccoidHelicobacter pylori. J Basic Microbiol 53: 147-155, 2013.

38. Feng Y, Wang L, Zeng J, et al: FoxM1 is overexpressed in Helicobacter pylori-induced gastric carcinogenesis and is negatively regulated by miR-370. Mol Cancer Res 11: 834-844, 2013.

39. Himaya SWA, Dewapriya P and Kim SK: EGFR tyrosine kinase inhibitory peptide attenuates Helicobacter pylori-mediated hyper-proliferation in AGS enteric epithelial cells. Toxicol Appl Pharmacol 269: 205-214, 2013. 